\title{
Nachrichten aus dem Zentralvorstand
}

Präimplantationsdiagnostik - Präimplantationsdiagnostik (PID) ist gemäss geltendem Fortpflanzungsmedizingesetz in der Schweiz verboten. Nach Annahme der Vorlage durch das Parlament soll sich dies nun in zwei Schritten ändern. Zuerst müssen die Stimmberechtigten über die Änderung von Art. 119 der Bundesverfassung am 14. Juni 2015 abstimmen. Wird diese Änderung angenommen, kommt die Änderung des Fortpflanzungsmedizingesetzes voraussichtlich Mitte 2016 zur Abstimmung. Dieser Abstimmungsvorlage empfiehlt der Zentralvorstand (ZV) zuzustimmen.

Aktionswoche Patientensicherheit - Die Stiftung Patientensicherheit Schweiz will im Rahmen einer Aktionswoche (14. bis 18. September 2015) der Patientensicherheit zu mehr Aufmerksamkeit in der Öffentlichkeit verhelfen und als ein ernstzunehmendes Thema im Bewusstsein der Gesellschaft etablieren. Gemeinsam mit Partnerorganisationen des Schweizer Gesundheitswesens durchgeführte Aktionen sollen aufzeigen, was zur Förderung der Patientensicherheit bereits getan wird und noch getan werden muss. Mit einem Sponsorenlauf könnte Geld für ein gemeinsam zu definierendes Projekt gesammelt werden. Der ZV stimmt zu, dass sich die FMH an der Aktionswoche beteiligt und entsprechende Aktivitäten plant.

Nationales Forschungsprogramm "End of Life» Für das Nationale Forschungsprogramm 67 «End of Life» untersucht die Gruppe Versorgungsforschung des Instituts für Sozial- und Präventivmedizin (ISPM) der Universität Bern die regionalen und zeitlichen Variationen in der Schweiz am Lebensende. Zu diesem Zweck fragt das ISPM die FMH für eine anonymisierte Datenlieferung $\mathrm{zu}$ den demographischen
Merkmalen der berufstätigen Ärzteschaft in der Schweiz an. Der ZV ist einverstanden, die Daten nach Unterzeichnung eines Datennutzungsvertrags zu liefern.

CHOP-Revision - Nachdem 2010 die Prozedurenklassifikation CHOP unter grossem Zeitdruck und nach unterschiedlichen Strukturen ausgeweitet wurde, ist nun eine Revision notwendig. Für die in Teilschritten geplante Überarbeitung hat das Bundesamt für Statistik (BFS) die FMH angefragt, als Bindeglied zwischen den Fachgesellschaften und dem BFS mitzuwirken und ihr Wissen einzubringen. Der ZV entscheidet, dass die FMH im Jahr 2015 die Antragsumsetzung/ CHOP-Revision (CHOP 2016) unterstützt und im Herbst 2015 die Weiterführung dieser Unterstützung für künftige CHOP-Revisionen prüft. Bis dahin kann sie Erfahrungen sammeln, ob sich ihre Rolle als Bindeglied bewährt hat und die Arbeiten und Aufgaben zwischen den Fachgesellschaften, dem BFS und der FMH klar abgegrenzt werden können.

Medizinprodukteverordnung - Die Medizinprodukteverordnung (MepV) regelt das Inverkehrbringen von Medizinprodukten und insbesondere die Bewertung ihrer Konformität. Mit der Übernahme der Durchführungsverordnung (EU) 920/2013 ins Schweizer Recht soll die Sicherheit von Medizinprodukten verbessert und die Anforderungen an Konformitätsbewertungsstellen harmonisiert werden. Damit wird zum einen ein gleich hohes Niveau des Patientenschutzes in der Schweiz wie in der EU sichergestellt, zum anderen aber auch, dass keine technischen Handelshemmnisse für die Schweizer Medizintechnikindustrie entstehen. Der ZV stimmt dem Stellungnahmeentwurf zu. 